\title{
Evidence-based medicine and complementary medicine
}

To apply evidence-based medicine (EBM) to complementary medicine (CM) - treatments such as acupuncture, chiropractic, homeopathy, or herbal medicine-seems, at first glance, a contradiction in terms. CM is often defined as techniques for which no evidence of benefit exists (or as a speaker at a recent Cochrane Colloquium put it, "medicine is either scientific or complementary"). But many of the interventions used by conventional clinicians have little scientific support; conversely, there is evidence from randomised trials in support of some interventions that are commonly described as CM. $\mathrm{CM}$ is an increasingly prevalent aspect of health care: about $10 \%$ of the UK population visit a practitioner each year, ${ }^{1}$ and about $40 \%$ of UK general practices offer their patients access to $\mathrm{CM}$ services on the national health service. ${ }^{2}$ As such, CM constitutes important area for the implementation of EBM for better patient care.

\section{Can EBM be applied to CM?}

There are two perspectives for thinking about EBM and CM. The external perspective is that of those outside CM. This might include a community mental health team deciding whether to employ an acupuncturist to treat addiction, a family physician considering whether to refer patients with anxiety disorders to a homeopath, or a patient deciding whether to buy an over the counter herbal remedy for depression. EBM can and should be used as part of such decisions. The internal perspective is that of CM practitioners themselves, finding, appraising, and acting on evidence to diagnose, treat, and prognose more effectively. This use of $\mathrm{EBM}$ is more problematic.

\section{EBM and decisions about referring to or purchasing CM}

Contrary to widespread belief, there is considerable evidence on which to base decisions about whether to refer to or purchase CM care. The registry of the Cochrane Collaboration field in CM currently lists over 4000 randomised controlled trials (RCTs), with a further 5000 papers awaiting analysis. There are about 200 systematic reviews of CM interventions, over 60 of which are protocols or completed reviews on the Cochrane Library. These deal with topics such as St John's Wort (a herbal treatment) for depression; acupuncture for migraine and chronic headache; osteopathy and chiropractic for neck pain; hypnosis for smoking cessation; and homeo pathy for influenza.

One problem with much CM research is that it often has low clinical relevance. For example, a well known RCT found significant differences between a homeopathic remedy and placebo for the treatment of hay fever. ${ }^{3}$ Normally, homeopaths give different remedies to patients with hay fever depending, at least in part, on non-medical characteristics of each patient such as their personality or taste in food. This makes homeopathy difficult-though not impossible-to test using standard RCT methodology. These researchers decided to use one remedy as an artificial "test case" because it allowed a simpler trial design. Their question was not: "is homeopathy an effective treatment for hay fever?," but: "is homeopathy a placebo?" The clinical implications of the study's findings for the management of hay fever are not straightforward therefore.

An associated problem is that the diseases and disorders which have been subject to CM research are not representative of those seen in clinical practice. There have been over 30 trials of acupuncture for nausea. ${ }^{4}$ This is not because nausea is a common presenting complaint in acupuncture, but because it is easy to research: only a single acupuncture point is used, large numbers of surgical patients can be recruited, and outcome can be assessed in a few hours. Chronic fatigue, a condition more commonly found in acupuncture practice, ${ }^{5}$ has not been evaluated, probably because such research presents considerably greater practical difficulties.

There are only a moderate number of RCTs of CM interventions in mental health. With the exception of certain herbal treatments such as St John's Wort for depression ${ }^{6}$ and kava-kava for anxiety, ${ }^{7}$ there are few high quality RCTs of any of the main CM treatments (acupuncture, homeopathy, herbal medicine, osteopathy, and chiropractic) for any of the main mental illness diagnoses. Even where mental health outcomes are assessed, most RCTs have not examined a mental health population. For example, although there are many trials showing a fall in anxiety scores after massage, most have studied acute anxiety associated with cancer, intensive care, or surgery. ${ }^{8}$

Another problem of implementing research in $\mathrm{CM}$ is that there are significant variations in the way that $\mathrm{CM}$ is practised. For any particular CM treatment there will be various different schools of thought with respect to methods of diagnosis and treatment. In acupuncture, for example, there are those who use traditional Chinese concepts of yin and yang and those who rely purely on Western neurophysiology. Among traditional acupuncturists, there are those who treat as the basis of the "five element" theory and those who treat using the "eight principles." Needles may be inserted deeply or so that they just pierce the skin, for a period of 30 seconds or 30 minutes, during which time they may (or may not) be stimulated by manual twirling, or with electricity. So, for example, an alcohol addiction unit considering referring patients for acupuncture might be impressed by an RCT retrieved from Medline, ${ }^{9}$ but may not know whether the acupuncture used by local practitioners is similar to that used in the trial.

Evidence-based decisions about purchasing or referring to CM may therefore require appraisal of research in which both the condition and the intervention are either unlike, or are not known to be like, the presenting problem. This should weaken, but not eliminate, any inferences made from clinical research. One of the strengths of the evidence-based medicine approach is that, through decision analysis, ${ }^{10}$ it provides a practical framework for the evaluation of CM evidence. CM treatments have long been prey to the view that no decision is worth taking until the evidence crosses a 
certain threshold (the level of which is never made explicit) at which point the evidence becomes "strong." Decision analysis, conversely, allows an explicit estimate of how much evidence is "enough" evidence.

In a simple decision tree with one decision (use or avoid treatment) and two outcomes (cure or illness), it can be shown that, to recommend treatment, the disutility of treatment must be lower than the disutility of the illness multiplied by the probability that the treatment will be successful. If a disease is considered to be 20 times worse than the risks, costs, and nuisance of a potential treatment, then that treatment is warranted once there is a greater than $5 \%$ chance that it will be effective. Many CM treatments are relatively safe, inexpensive, and non-invasive, and therefore have low disutilities. The wider use of such analyses may result in such decisions that, although homeopathy should not be a routine option in depression, there is a case for referring cases where the response to conventional treatment has been poor or when drug adverse effects are causing particular distress.

\section{Use of EBM by CM practitioners}

Considerable barriers remain to the use of EBM by practitioners of CM. The main problem is a dearth of research data examining those questions most likely to be asked by practitioners in the course of their work. So although evidence exists that chiropractors can offer effective treatment in back pain, ${ }^{11}$ there is less evidence on which types of spinal manipulation are most beneficial. Similarly, the benefits of acupuncture for migraine have been supported by RCTs,${ }^{12}$ but there is no information on which strategies forpoint selection should be favoured, or how long needles should be kept in place or how many treatment sessions are optimal. Undertaking and implementing such research would require a greater commitment to science, critical thinking, and EBM than is currently found in the CM community.

ANDREW VICKERS, DPhil Integrative Medicine Service, Memorial Sloane-Kettering Cancer Center, New York, NY, USA

1 Vickers A. Use of complementary therapies. BMJ 1994;309:1161.

2 Thomas K, Fall M, Parry G, et al. National survey of access to complementary health care via general practice. Sheffield: SCHARR, 1995. Copies available from Medical Care Research Unit, SCHARR Regent Court, 30 Regent Street, Sheffield S

3 Reilly DT, Taylor MA, McSharry C, et al. Is homeopathy a placebo response? Controlled trial of homoeopathic potency, with pollen in hayfever as model. Lancet 1986;ii:881-6.

4 Vickers AJ. Can acupuncture have specific effects on health? A systematic review of acupuncture antiemesis trials. $J R$ Soc Med 1996;89:303-11.

5 Wadlow G, Peringer E. Retrospective survey of patients of practitioners of traditional Chinese acupuncture in the UK. Complementary Therapies in Medicine 1996:4:1-7.

6 Linde K, Ramirez G, Mulrow CD, et al. St John's Wort for depression-an overview and meta-analysis of randomised clinical trials. BMJ 1996;313: 253-8.

7 Volz HP, Kieser M. Kava-kava extract WS 1490 versus placebo in anxiety disorders - a randomized placebo-controlled 25-week outpatient trial. Pharmacopsychiatry 1997;30:1-5.

8 Vickers AJ. Massage and aromatherapy: a guide for health professionals. London: Chapman and Hall, 1996. Reprinted as: Cheltenham: Stanley Thornes, 1998.

9 Bullock ML, Culliton PD, Olander RT. Controlled trial of acupuncture for severe recidivist alcoholism. Lancet 1989;i:1435-9.

10 Weinstein MC, et al. Clinical decision analysis. Philadelphia: WB Saunders, 1980 .

11 Meade TW, Dyer S, Browne W, et al. Randomised comparison of chiropractic and hospital outpatient management for low back pain: results from extended follow up. BMJ 1995:311:349-51.

12 Vincent CA. A controlled trial of the treatment of migraine by acupuncture. Clinical Journal of Pain 1989;5:305-12.

Modified version also published in Evidence-Based Medicine.

\section{EBMH feedback on the web}

We welcome letters from our readers about Evidence-Based Mental Health. Your feedback is most important in assisting us to produce a high quality journal which is useful to the practising mental health clinician. All letters will be put up on the web site; for the latest feedback please go to http://www.psychiatry.ox.ac.uk/cebmh/journal/letters.html

All letters should be typewritten, double spaced, and mailed or faxed to Nancy Wilczynski in the Canadian editorial office (Health Information Research Unit, Faculty of Health Sciences, McMaster University, HSC 3H7, Hamilton, Ontario L8N 3Z5, Canada. Fax + 1905546 0401).

\section{How to cite Evidence-Based Mental Health}

CITATION OF MATERIAL FROM THE NOTEBOOK:

- Streiner D, Geddes J. Some useful concepts and terms used in articles about diagnosis [EBMH notebook]. Evidence-Based Mental Health 1998 Feb;1:6-7.

CITATION FOR MATERIAL TAKEN FROM A STRUCTURED ABSTRACT, WRITTEN WITHOUT ATTRIBUTION BY A STAFF MEMBER:

- Caregiver training delayed admission of patients with dementia to nursing homes [abstract]. Evidence-Based Mental Health 1998 Feb;1:9. Abstract of: Brodaty H, Gresham M, Luscombe G. The Prince Henry Hospital dementia caregivers' training programme. Int J Geriatr Psychiatry 1997 Feb;12:183-92.

CITATION FOR MATERIAL TAKEN FROM A COMMENTARY TO AN ARTICLE:

- Bradley C. Commentary on "Purchase of a handgun was associated with an increased risk of suicide or death by homicide." Evidence-Based Mental Health 1998 Feb;1:28. Comment on: Cummings P, Koepsell TD, Grossman DC, et al. The association between the purchase of a handgun and homicide or suicide. Am J Public Health 1997 Jun;87:974-8.
} 